\title{
Who influences parents' decision on life support treatments for children with spinal muscular atrophy type 1 ?
}

Fermín Jesús González-Melado ${ }^{1 *}$, Vincenzo Maria Di Ciommo ${ }^{2}$, Maria Luisa Di Pietro ${ }^{1,3}$, Chiara Mastella ${ }^{4}$, Maria Beatrice Chiarini Testa ${ }^{2}$ and Renato Cutrera ${ }^{2}$

${ }^{1}$ Pontifical John Paul II Institute for studies on Marriage and Family, Rome, Italy

${ }^{2}$ Bambino Gesù Children's Hospital IRCCS, Rome, Italy

${ }^{3}$ Catholic University of the Sacred Heart, Rome, Italy

${ }^{4}$ Fondazione IRCCS Ca' Granda, Ospedale Maggiore Policlinico, Milan, Italy

\begin{abstract}
Background: We aimed at assessing the influence of those who are part of the parents' social network (family, friends and health professionals) when they are making decisions about nutritional and respiratory support treatments for their SMA 1 children.

Methods: We prepared a 46-question questionnaire to explore the factors that influence parents' decisions. The influence of the different persons, the degree of satisfaction with the treatment elected and the consistency between the treatment information and the child's status were assessed by the parents using a Likert scale of ten (a higher score for a higher influence).
\end{abstract}

Results: The secondary-care physicians have the main influence on parent's decisions about nutritional support (mean=6.4) and about respiratory support (mean=5.8). This influence is higher among decisions about Percutaneous Endoscopic Gastrostomy (mean=8.0) and among decisions about Tracheostomy Ventilation (mean=8.0). The degree of satisfaction with the chosen treatment is high (mean=7.9), and consistency between information about treatment and development of the illness also has a high score (mean=8.3). The hospital ethics committee was involved in $13.3 \%$ of the cases.

Conclusions: The secondary-care physician influences parents' decisions about nutritional and respiratory support, particularly while choosing PEG and TV. The degree of satisfaction is very high regardless of the treatment chosen. The hospital ethics committee is not usually involved in the treatment decisions for SMA1 children.

\begin{abstract}
Abbreviations: LST - Life support treatments; NRA - continuous non-invasive respiratory muscle aid; PEG - percutaneous endoscopic gastrostomy; SMA - Spinal Muscular Atrophy; SMA1 - Spinal Muscular Atrophy type 1; TV - tracheostomy and invasive mechanical ventilation

\section{Introduction}

Spinal muscular atrophy (SMA) is the second most common, potentially lethal autosomal recessive disorder [1]. This disorder affects 1 in 8.000 to 10.000 infants with a carrier frequency of 1 in 34 [2]. Children with SMA type 1 can never roll, sit, or walk [3]. SMA1 is a progressive disease [4,5], and is usually fatal in infancy [6]. Parents of children with SMA1 have three options for respiratory treatment: letting nature take its course; tracheostomy and invasive mechanical ventilation (TV); continuous non- invasive respiratory muscle aid (NRA), including non-invasive ventilation and mechanically assisted cough [7]. NRA and TV can prolong survival, with NRA showing a lower survival probability at ages 24 and 48 months [8].
\end{abstract}

There are many studies that examine physicians' preferences in managing treatments, their attitudes with regard to a particular disease or to specific decisions such as withholding or withdrawing life support treatments (LST) [9-11]. However, there are not so many studies examining the people that influence parents' decisions about the choice of a specific treatment for their children [12]. These studies show that parents take decisions in a social context that change over time and that is influenced by relationships with family, friends and health professionals [13]. Also, these studies valued information that parents receive as a fundamental part of a decision-making process, and they try to measure the degree of parental satisfaction with that decision. We wanted to assess the influence of those who are part of the parents' social network (family, friends and health professionals) when they are making decisions about nutritional and respiratory support treatments. We also wanted to assess the parents' degree of satisfaction with the decisions taken and the correspondence between information received from the physicians and the medical condition of the child.

*Correspondence to: Fermín Jesús González-Melado, Pontifical John Paul II Institute for Studies on Marriage and Family, Lateran University, Piazza San Giovanni in Laterano, 4 - 00120 Vatican City, Rome, Italy, Tel: +34 630-438-796; Fax: +34 924221 245; E-mail : ferminjgm@hotmail.com

Key words: life support treatments, spinal muscular atrophy type 1, nutritional support, respiratory support, parents

Received: January 03, 2016; Accepted: January 28, 2016; Published: February 02,2016 


\section{Methods}

\section{Subjects of our study}

Data from the subjects of our research were extracted from the databases of the Bambino Gesù Children's Hospital (Rome), SAPRE - UONPIA - Fondazione IRCCS Ca' Granda, Ospedale Maggiore Policlinico (Milan), and the families' associations (the Associazione Federica, Associazione FamiglieSMA and Associazione per lo Studio delle Atrofie Muscolare Spinali Infantile-ASAMSI). All participants had to fulfill two inclusion criteria: a) to be parents of at least one child diagnosed with SMA1 and; b) SMA1 child's diagnosis had been genetically confirmed. Three hundred and one patients fulfilled inclusion criteria and questionnaires were posted to their parents. The postal service returned 13 of them that were undelivered because the address was wrong or people had moved to another town. The data from a family was discarded because the child had a spinal muscular atrophy with respiratory distress and thus did not fulfill the selection criteria of our research, and our target population was made up of 287 patients. At the end of June 2013, 124 participants had responded. Three questionnaires had more than half of the questions unanswered and therefore were considered invalid. The population number has been reduced to 121 valid responses. (response rate $=42.16 \%$ ).

\section{The questionnaires}

The research was conducted providing questionnaires to parents by postal delivery. One single questionnaire with 46 scaled questions about motivations for treatment choice was sent to all the parents (see Appendix 1). The questions were identical for both parents with children SMA1 alive and those parents of deceased SMA1 children. The questions were divided into seven sections: general information about the child, about the brothers and sisters, disease information, information about Mum, about Dad, sociological data and questions related to the parents' decision process regarding the treatment. This last section included questions about the people that influence parents' decision on nutritional and respiratory support. The questions about the influence of the different persons over the decision process of treatment, the degree of satisfaction with the treatment elected, and the correspondence between treatment information and the child's status, were answered by parents assessing a score in a scale of ten (a higher score for a higher influence).

The content of the postal envelope that parents received at home was:

-A letter presenting our study.

-The questionnaire with 46 scaled questions.

-A pre-posted envelope to return the questionnaire, addressed to the Respiratory Medicine Service of the Bambino Gesù Children's Hospital, was included.

The questionnaires were posted during March and June of 2012 and collected until June 2013. No incentive was offered for completion of the questionnaire.

Statistical analysis: Results are reported as means. Inferences are calculated by two groups of comparison tests (2-side cues); when the groups were $>2$ ANOVA was applied, with a post-hoc Bonferroni test. Statistical software SPSS was used; a $\mathrm{p}<0.05$ was considered statistically significant.

Ethical Issues: Our survey had been evaluated and approved by the Ethics Committee of the Bambino Gesù Children's Hospital (RomeItaly) on September 15th, 2011.

\section{Results}

\section{Coping the families of SMA1 children}

There was a $51.2 \%$ of the questionnaires were only answered by the mother $(51,2 \%)$; while $9,1 \%$ of them have only been fulfilled by the father. With the rest of the cases the questionnaires were completed jointly by the father and the mother $(38.8 \%)$ and only $0.8 \%$ of cases were filled in by another person.

The child with SMA 1: Sex distribution shows that $52.9 \%$ of patients were males and $47.1 \%$ females. $62.8 \%$ of patients were born spontaneously while in $37.2 \%$ of cases it was necessary to perform a caesarean section.

Diagnosis occurred in most cases within 6 months (80.2\%), between 6 and 12 months in $18.2 \%$ of cases and $1.7 \%$ of cases after the first year of life. In $66.1 \%$ of cases the child had died at the time of completion of the questionnaire. In $76.3 \%$ of deceased patients cases death occurred before the age of one; $11.3 \%$ had died between the age of one and two. The remaining $12.5 \%$ deaths had occurred after two and half years.

Regardless of respiratory support, the number of hospitalizations is very low and in most cases (84.3\%) less than 5 times, and only $5 \%$ of cases more than 11 times during the lifetime of the child. This low number of hospitalizations is due to improved disease management at home by the families and primary care health workers.

The parents: The mother is an Italian woman, whose average age at the time of SMA 1 child birth was 31.2 years old. In most cases (43.0\%) she has a high school degree; she is working at the time of the survey (52.1\%), although at the time of diagnosis of the child's illness she had to change her work situation $(66.0 \%)$. This change meant to leave her work in order to care for the sick child (39.7\%) or to reduce her working hours $(25.0 \%)$. Who normally cares for the child? In $95 \%$ of cases the mother is present for the responses, with $58.3 \%$ of cases displayed as the only career of the child.

The father is Italian (94.2\%) and he was about 34.4 years old at the time of the birth of the sick child. Less than a half of fathers have high school studies (43.8\%). Currently, he is working (91.7\%), and at the time of the child's illness, in most of cases but less than their wives, changed their employment status (58.8\%); the change was a reduction in working hours (51.4\% of those who changed their status). Although the father participates in child care either with the mother $(31.7 \%)$ or with the mother and another person (5.0\%), the fact is that in only $2.5 \%$ of cases the father appears exclusively as the person who normally cares for the sick child.

Before the disease $100 \%$ parents live together while after coping face with the illness this situation is reduced to $94.2 \%$. Of those who do not live together (5.8\%), $4.1 \%$ are divorced, $0.8 \%$ singles and $0.8 \%$ separated. These results are important if we consider that in the general Italian population there was a $1.81 \%$ divorce rate and $3.0 \%$ separation rate [14]. This means that couples with a SMA1 child have a rate of divorce two times higher than the general population.

The brothers and sisters: Most families had two or more children, including the child with SMA1 (88.1\%). From them, $66.7 \%$ of parents responded that the brothers and sisters were acquainted about the disease. The main effects detected were anxiety and worry. A higher level of responsibility is also one of the effects of the disease across the siblings (Figure 1). 


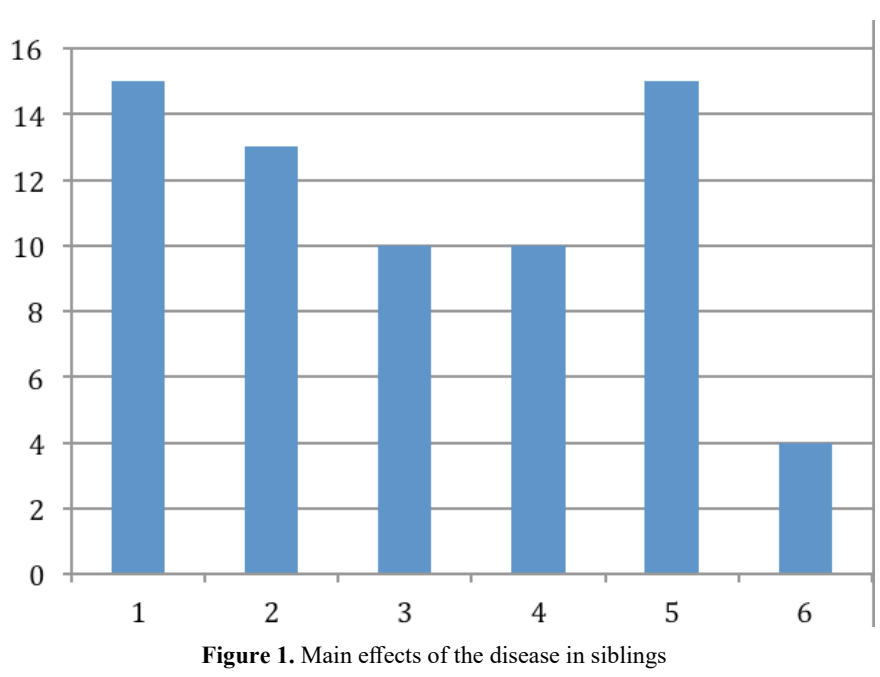

$1=$ more anxious and worried; $2=$ most in need of affection; $3=$ more angry and undisciplined; $4=$ more cuddly; $5=$ more responsible for; $6=$ other

\section{Treatment decisions}

\section{Treatments chosen}

Exactly a half of the patients had been fed only by a nasogastric tube during the development of the disease; $31.7 \%$ had used also percutaneous endoscopic gastrostomy (PEG), while only $18.3 \%$ were fed exclusively by the oral route. From the data collected, $17.3 \%$ of parents used TV; $40.8 \%$ used NRA and $41.8 \%$ chose NT.

\section{People that influence treatment decisions}

The secondary-care physician, i.e. pulmonologist or neurologist, (mean score $=6.4$ points out of 10 ) has the most influence on the parents' decision about nutritional support (Figure 2). This is most clearly in the decision to place the PEG where the degree of influence of the secondary-care physician increases to values of 8.0 points. The pediatrician (2.8 points) and section reserved for other people $(2.3$ points) are those who, after secondary-care physicians, have more influence on the parents' decision.

The PEG was influenced, as expected, by the secondary-care physicians as compared with the oral route (mean 8.0 versus 5.0, $\mathrm{p}=0.02$ ) and by relatives without medical knowledge when it was compared with nasogastric tube (with marginal significance only, 2.3 versus $0.93, \mathrm{p}=0.05$ ). No difference was found in the other groups of potentially advisors.

With regard to who has more influence during the parents' decision process about ventilation, the secondary-care physician also appears as the most influential with a mean score $=5.8$ points.

The influence of the secondary-care physician is greater when choosing TV, which increases to 8.1 points, while less influence when they decide to not provide ventilatory support with only 3.2 points out of 10 . The pediatrician and other people have less influence during the decision process of respiratory support (Figure 3).

The degree of satisfaction and the adequacy on treatment information

The degree of satisfaction with the decision on the form of nutrition is high (mean score $=7.9$ points out of 10 ) and consistency between the information received and present conditions of the child (mean score $=8.3$ points out of 10 ) is also high. These results are significant when comparing the degree of satisfaction with the form of nutritional support: oral nutrition, nasogastric tube and PEG (Figure 4a), with no significant difference between the three forms. The same is true when studying the adequacy on treatment information regarding the nutritional support (Figure 4b).

This data remains when we analyze the degree of parental satisfaction about the ventilatory support decision, which is high $($ mean $=7.9$ and median $=10)$. There is a significant difference $(p=0.003)$ in interquartile range ( 3 for TV; 2 for NRA; 1,25 for NT) (Figure 4c). Also the scores about the correspondence between information given by physicians regarding ventilation and the children status are high ( mean=7.8 and median=10). There is a significant result when studying the adequacy on treatment information for the type of respiratory support: TV, NRA and NT (Figure 4d), with a significant difference $(\mathrm{p}=0.006)$ that shows a minor interquartile rank for $\mathrm{NT}=1$ and a higher interquartile rank for NRA and for TV ( 3 for NRA and 4 for TV).

When we carefully studied the low score extreme cases, we saw that some cases correspond with situations in which the parents had not participated in the decision process about the nutritional and respiratory support treatment for their child (Figure 4).

\section{Participation of the Hospital Ethics Committee}

On a sample size of $n=105$, parents said that the hospital ethics committee was not involved in the decision making process $(47.6 \%)$ and only $13.3 \%$ of responses said that the committee participated in it. A considerable proportion (39\%) did not know if the committee had been involved in the decision making process. These responses show that hospital ethics committees are not usually involved in decisions about LST for SMA1 children.

\section{Discussion}

\section{Who influences the treatment decision process?}

We observed that the means of the influence of the various potential advisors are very low and almost insignificant, except for the secondary-care physician. Some data may be subject to closer scrutiny; i.e. the little influence of the family on parents when they decided to not

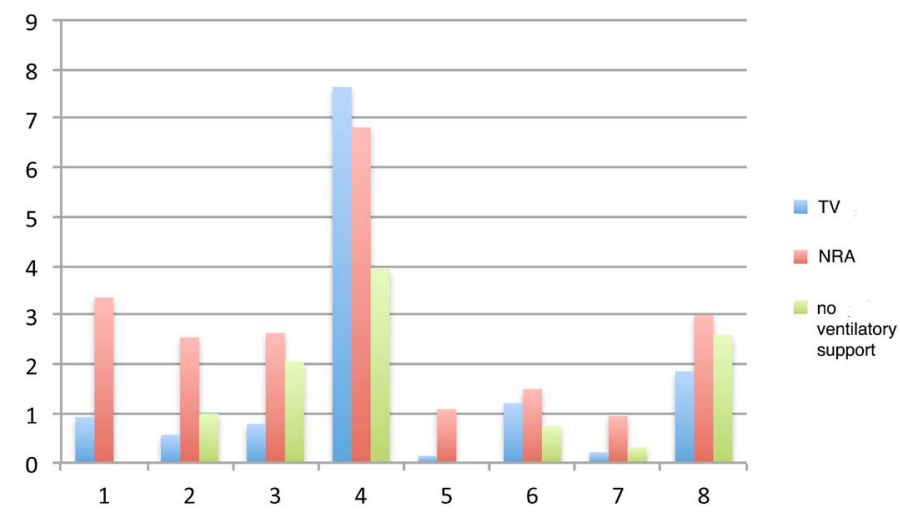

Figure 2. Question D.40, Rating of different people's influence during parents` decision process about type of nutrition

The graph shows the value that parents give to the influence of others during the decision process about the kind of nutrition: First column $=$ oral nutrition; second column=nasogastric tube and third column=PEG. People that can influence: $1=$ Familiar without any medical knowledge; $2=$ familiar with medical knowledge; $3=$ Pediatrician; 4=Secondary-care physician; $5=$ Friends without any medical knowledge; $6=$ Friends with medical knowledge $7=$ Spiritual guide; and $8=$ Other people (usually SMA associations or institutions that attend children and parents' necessities.) 


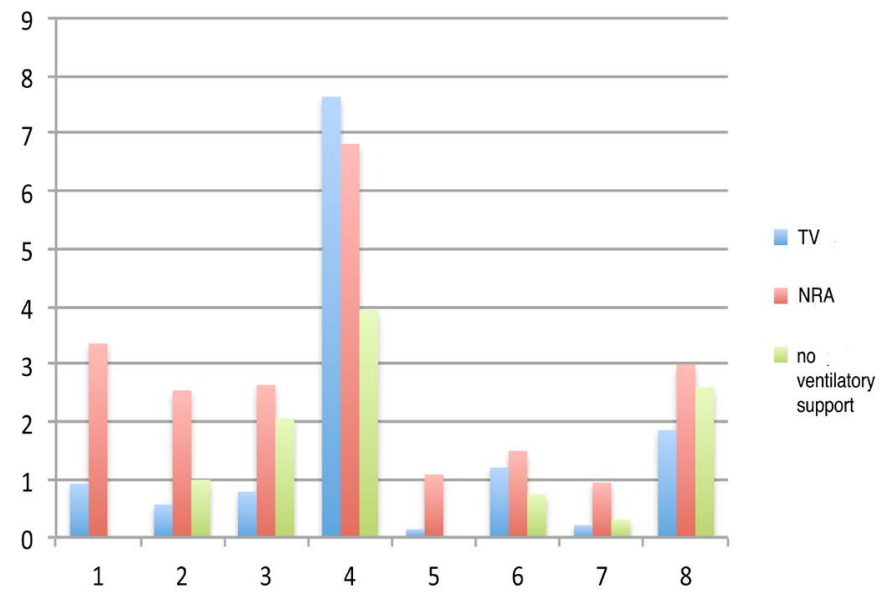

Figure 3. Question D.43. Rating different people's influence during parents' decision process about type of ventilator support

The graph shows the value that parents give to the influence of others during the decision process about the type of ventilatory support: First column=invasive ventilation; Second column=noninvasive ventilation and third column=to not provide ventilatory support. People that can influence: $1=$ Familiar without any medical knowledge; $2=$ familiar with medical knowledge; $3=$ Pediatrician; 4=Secondary-care physician; $5=$ Friends without any medical knowledge; $6=$ Friends with medical knowledge; $7=$ Spiritual guide; and $8=$ Other people (usually SMA families associations or institutions that attend children and parents' necessities.)

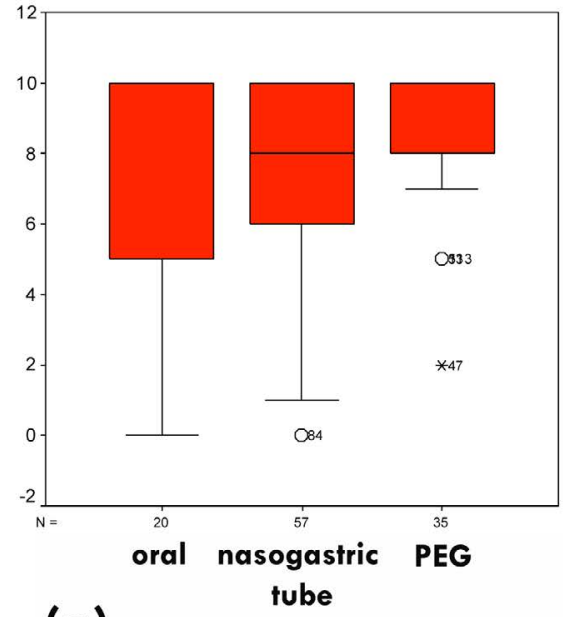

(a)

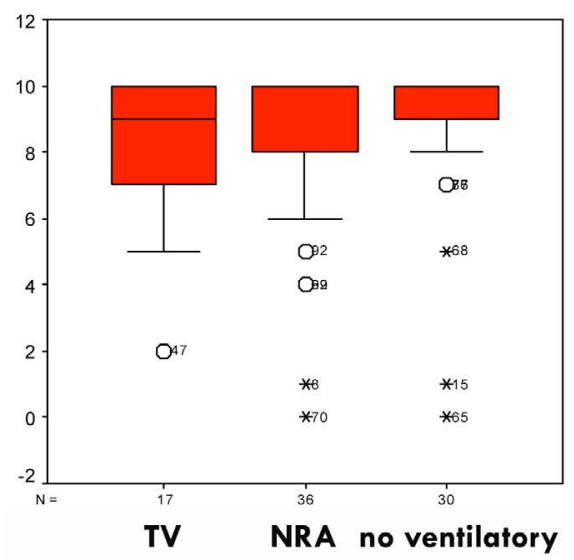

(c)

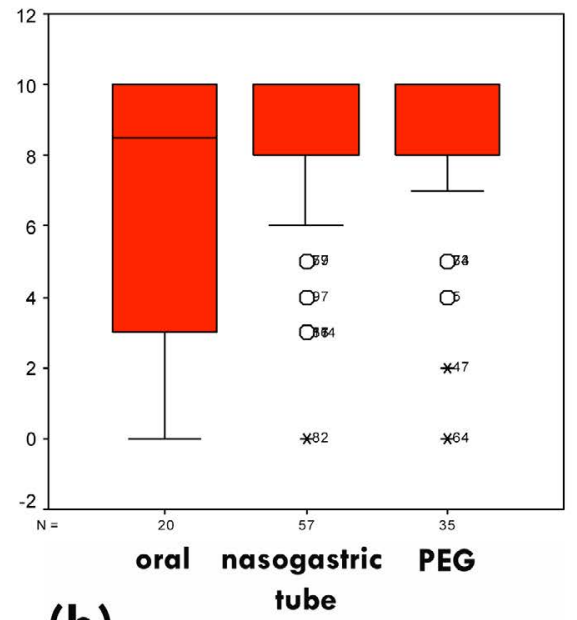

(b)

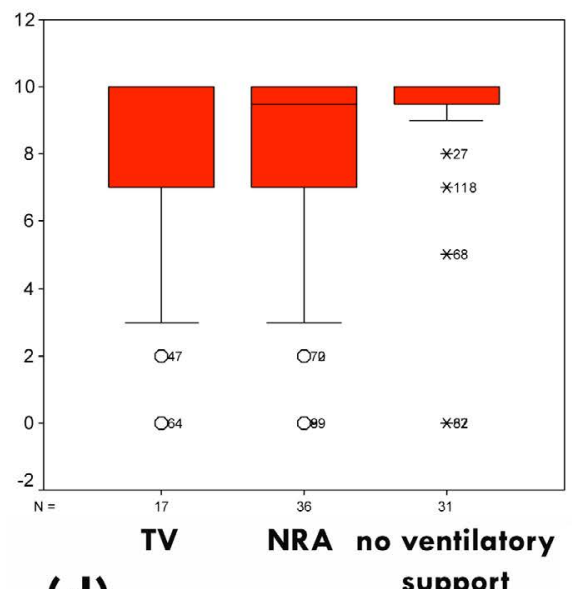

(d)

Figure 4. Parents' satisfaction with nutritional and ventilator support, and adequacy between information about the treatments and the child's status. a) Parents' satisfaction regarding nutritional support decision. b) Correspondence between information about nutritional support and the condition of the child. c) Parents' satisfaction regarding ventilatory support decision. d) Correspondence between information about ventilatory support and the child status.

Some extreme low score cases correspond with situations in which the parents do not participate in the decision process of treatment: Case 47 corresponds to a child who was intubated during a respiratory crisis, without the parents' consent. In case 50 the parents could not choose the treatment and was the secondary-care physicians who decided NT for their child. Case 64 corresponds to a child whose parents had to face doctors in order to choose TV for their child. 
give ventilatory support. The parents know that the decision to deny ventilatory support is an option that limits the life expectancy of the child and that this decision is made relying on the medical expertise of the specialists and the experience of other parents with SMA1 children.

Ventilation (TV or NRA) was influenced more than NT when the secondary-care physicians were examined (NT mean $=3.2$ versus, respectively TV mean $=8.1$ and NRA mean $=7.1, \mathrm{p}<0.001)$, while relatives or friends without medical knowledge influenced NRA versus $\mathrm{NT}$ (relatives NRA mean $=2.4$ versus NT mean $=0.4$, and friends NRA mean $=1.3$ versus NT mean $=0.0$, in both cases $\mathrm{p}=0.01$ )

These results are important if we consider the last international survey on physician / tracheostomy SMA1 ratio revealed that only $60 \%$ of respondents considered medical TV as an acceptable treatment for SMA1 children who had chronic respiratory failure [17], while in the same survey, most physicians (86\%) favor long-term NRA in SMA1 children.

Only $51 \%$ of physicians surveyed by Benson knew the new treatment protocols approved for the SMA1 children [17]. If medical experts do not know the protocols then, on what basis do they recommend treatment? Recommendations are based on their experience with SMA1 children with TV. Physicians with SMA1 TV patients are more likely to recommend TV to future patients, while those without experience with SMA1 TV patients are less inclined to recommend TV [16-17,20]. This is important to the education of secondary-care physicians in the treatment protocols approved by the various international committees for children with SMA1 [33].

\section{The degree of satisfaction with decisions about treatments}

We have seen that parental satisfaction is very high regarding their decisions of treatments for respiratory and nutritional support. This data is consistent with that obtained in other studies [12]. Some extreme low score cases corresponded with situations in which the parents had not participated in the decisions process about treatment (Figure 4). The exclusion of families in the decision process is contrary to disease management protocols [21,22]. Dissatisfaction is generated when the family preferences are not taken into account.

What is the correspondence between the information provided by physicians and the medical condition of the child?

As for the correspondence between the information received and the child's development the results are also very high. Other studies show high parental satisfaction with the information received [13]. The information received is one of the factors that parents considered fundamental when deciding, valuing information that is clear and appropriate to the evolution of the child's illness [12,15]. Protocols emphasize the necessity to give all treatment options to parents with full and clear information [21-23], and this is what parents demand [15]. Doctors must avoid the paternalistic attitudes in hiding information about possible treatments, for example, long-term TV [24]. It is clear that parents cannot make decisions about accepting a treatment or not for their SMA1child unless the doctor told them the truth about child's condition and its evolution [24]. Access to information on the Internet makes parents to question the doctors' approach when they do not offer all the possible options of treatment.

\section{Statistical weakness}

On the one hand, our study achieved a respectable size $(n=121)$ compared to other studies about parents of children with SMA1 [1819]. But, on the other hand, the weakness of our sample prevented us from doing a factor analysis to firmly establish associations with other factors such as geographic location or parents' religious status. Our results are limited to a country (Italy) with a specific cultural context. For this reason, our data should serve as a starting point to larger and statistically more significant studies to corroborate or refute the analysis carried out.

\section{Retrospective nature of our motivational study}

Our research is a retrospective study. This means that we questioned parents about a decision that they made in the past. Such methodology implies a pre-conceived idea about what kind of people could influence the parents during the decision-making process. In addition, this methodology requires from parents the effort to collect and also of organize their thoughts and feelings, and bring them from the past into the present.

\section{Conclusions}

The secondary-care physician (pulmonologist or neurologist) has the most influence on the parents' decisions concerning nutritional and respiratory support. This also on influence is even clearly manifested when choosing PEG and when choosing TV. The degree of satisfaction of the decision is very high in both (nutritional and respiratory support), regardless of the option chosen for respiratory and nutritional status. Extremely low cases, in terms of satisfaction, are related not to the type of ventilation or nutrition, but with no family involvement during the treatment decision process, regardless of the decision taken. The correspondence between the treatment information provided by the medical team and the evolution of the disease is very high. The ethics committees of the hospital are not usually involved in treatment decision process for SMA1 children.

\section{Funding source}

This research was supported by the Archdiocese of Mérida-Badajoz (Spain).

\section{Financial disclosure statement}

No honorarium, grant, or other form of payment was given to any of the authors to produce this manuscript.

\section{Conflict of interests}

The authors declare that they do not have competing interests in this research. Each author has seen and approved the submission of this version of the manuscript and takes full responsibility for the manuscript.

\section{Contributors}

Fermín J. González-Melado conceptualized and designed the study, designed the questionnaire, coordinated data collection, supervised statistical results, drafted the initial manuscript, and approved the final manuscript as submitted.

Vincenzo M. Di Ciommo designed the data collection instruments, analyzed the statistical results, critically reviewed the manuscript, and approved the final manuscript as submitted.

Maria Luisa Di Pietro, Chiara Mastella, Maria B. Chiarini-Testa, R. Cutrera carried out the initial analyses and supervised data collection. All of them reviewed and revised the manuscript, and approved the final manuscript as submitted. 


\section{Acknowledgements}

Thanks to all of the SMA1 families who participated in our study. This research was supported by the Archdiocese of Mérida-Badajoz (Spain).

\section{References}

1. Ogino S, Wilson RB (2004) Spinal muscular atrophy: molecular genetics and diagnostics. Expert Rev Mol Diagn 4: 15-29. [Crossref]

2. Swoboda KJ, Prior TW, Scott CB, McNaught TP, Wride MC, et al. (2005) Natural history of denervation in SMA: relation to age, SMN2 copy number, and function. Ann Neurol 57: 704-712. [Crossref]

3. Kolb SJ, Kissel JT (2011) Spinal muscular atrophy: a timely review. Arch Neurol 68 : 979-984. [Crossref]

4. Burghes AH, Beattie CE (2009) Spinal muscular atrophy: why do low levels of survival motor neuron protein make motor neurons sick? Nat Rev Neurosci 10: 597 609. [Crossref]

5. Wirth B, Brichta L, Hahnen E (2006) Spinal muscular atrophy: from gene to therapy. Semin Pediatr Neurol 13: 121-131. [Crossref]

6. Lemoine TJ, Swoboda KJ, Bratton SL, Holubkov R, Mundorff M, et al. (2012) Spinal muscular atrophy type 1: are proactive respiratory interventions associated with longer survival? Pediatr Crit Care Med 13: e161-165. [Crossref]

7. Oskoui M, Levy G, Garland CJ, Gray JM, O'Hagen J, et al. (2007) The changing natural history of spinal muscular atrophy type 1. Neurology 69: 1931-1936. [Crossref]

8. Gregoretti C, Ottonello G, Chiarini Testa MB, Mastella C, Ravà L, et al. (2013) Survival of patients with spinal muscular atrophy type 1. Pediatrics 131: e1509-1514. [Crossref]

9. Levetown M, Pollack MM, Cuerdon TT, Ruttimann UE, Glover JJ (1994) Limitations and withdrawals of medical intervention in pediatric critical care. JAMA 272: 12711275. [Crossref]

10. Randolph AG, Zollo MB, Egger MJ, Guyatt GH, Nelson RM, et al. (1999) Variability in physician opinion on limiting pediatric life support. Pediatrics 103: e46. [Crossref]

11. Burns JP, Mitchell C, Griffith JL, Truog RD (2001) End-of-life care in the pediatric intensive care unit: attitudes and practices of pediatric critical care physicians and nurses. Crit Care Med 29: 658-664. [Crossref]
12. Meert KL, Thurston CS, Sarnaik AP (2000) End-of-life decision-making and satisfaction with care: parental perspectives. Pediatr Crit Care Med 1: 179-185. [Crossref]

13. Meyer EC, Burns JP, Griffith JL, Truog RD (2002) Parental perspectives on end-of-life care in the pediatric intensive care unit. Crit Care Med 30: 226-231. [Crossref]

14. ISTITUTO NAZIONALE DI STATISTICA. Separazioni e divorzi in italia. Statistiche Report 2012: 1-15.

15. Sharman M, Meert KL, Sarnaik AP (2005) What influences parents' decisions to limit or withdraw life support? Pediatr Crit Care Med 6: 513-518. [Crossref]

16. Hardart MK, Burns JP, Truog RD (2002) Respiratory support in spinal muscular atrophy type I: a survey of physician practices and attitudes. Pediatrics 110: e24. [Crossref]

17. Benson RC, Hardy KA, Gildengorin G, Hsia D (2012) International survey of physician recommendation for tracheostomy for Spinal Muscular Atrophy Type I. Pediatr Pulmonol 47: 606-611. [Crossref]

18. Meldrum C, Scott C, Swoboda KJ (2007) Spinal muscular atrophy genetic counseling access and genetic knowledge: parents' perspectives. J Child Neurol 22: 1019-1026. [Crossref]

19. Chung BH, Wong VC, Ip P (2004) Spinal muscular atrophy: survival pattern and functional status. Pediatrics 114: e548-553. [Crossref]

20. Hardart MK, Truog RD (2003) Spinal muscular atrophy--type I. Arch Dis Child 88 848-850. [Crossref]

21. Hull J, Aniapravan R, Chan E, Chatwin M, Forton J, et al. (2012) British Thoracic Society guideline for respiratory management of children with neuromuscular weakness. Thorax 67 Suppl 1: i1-40. [Crossref]

22. Wang CH, Finkel RS, Bertini ES, Schroth M, Simonds A, et al. (2007) Participants of the International Conference on SMA Standard of Care. Consensus statement for standard of care in spinal muscular atrophy. J Child Neurol 22: 1027-1049.

23. Roper H, Quinlivan R (2010) Workshop participants. Implementation of "the consensus statement for the standard of care in spinal muscular atrophy" when applied to infants with severe type 1 SMA in the UK. Arch Dis Child 95: 845-849.

24. Oñate E, Límites de la ventilación no invasiva en niños, in Esquinas A, ed. Bioética en ventilación mecánica no invasiva. Fundamentos, Ergon, Madrid 201: 97-105.

Copyright: (C2016 González-Melado FJ. This is an open-access article distributed under the terms of the Creative Commons Attribution License, which permits unrestricted use, distribution, and reproduction in any medium, provided the original author and source are credited. 